Conf- $9410207-1$

UCRL-JC-117582

PREPRINT

\title{
Determination of the Concentration and Isotopic Composition of Uranium in Environmental Air Filters
}

\author{
RECEVED \\ OCT 061994 \\ OSTI
}

\author{
G. Price Russ III \\ Jeanne M. Bazan
}

This paper was prepared for submittal to the

Symposium on Applications of ICP-MS to

Radionuclide Determinations

Gatlinburg, TN

October 11-13, 1994

August 26, 1994
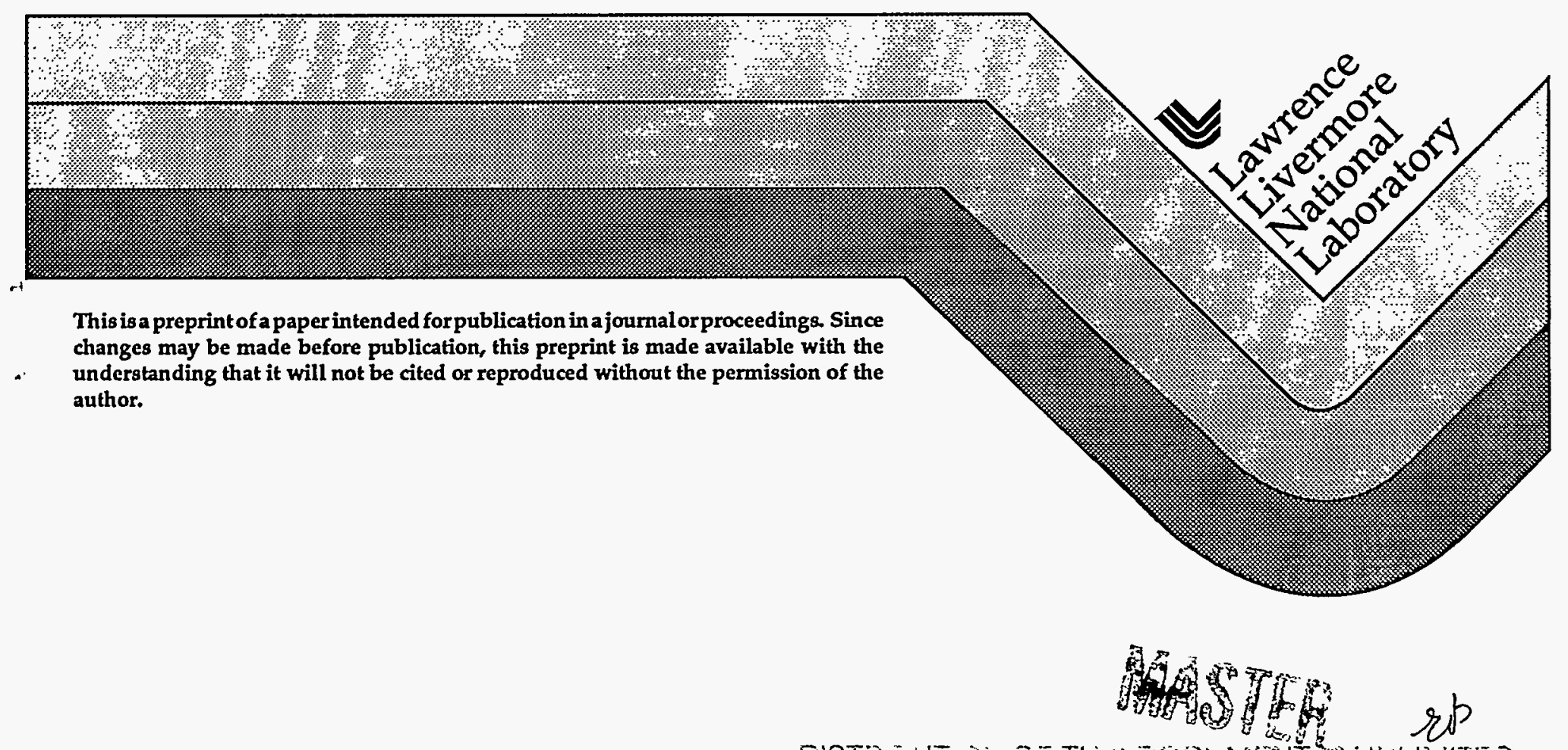


\section{DISCLAIMER}

This report was prepared as an account of work sponsored by an agency of the United States Government. Neither the United States Government nor any agency thereof, nor any of their employees, make any warranty, express or implied, or assumes any legal liability or responsibility for the accuracy, completeness, or usefulness of any information, apparatus, product, or process disclosed, or represents that its use would not infringe privately owned rights. Reference herein to any specific commercial product, process, or service by trade name, trademark, manufacturer, or otherwise does not necessarily constitute or imply its endorsement, recommendation, or favoring by the United States Government or any agency thereof. The views and opinions of authors expressed herein do not necessarily state or reflect those of the United States Government or any agency thereof. 


\section{DISCLAIMER}

Portions of this document may be illegible in electronic image products. Images are produced from the best available original document. 
TITLE OF sYMPOSIUM: Applications of Inductively Coupled PlasmaMass Spectrometry (ICP-MS) to Radionuclide Determinations

\section{AUTHORS' NAMES:}

G. Price Russ $\operatorname{III}^{1}$ and Jeanne M. Bazan ${ }^{2}$

\section{TITLE OF PAPER:}

Determination of the Concentration and Isotopic composition of Uranium in Environmental Air Filters

\section{AUTHORS ' AFFILIATION:}

${ }^{1}$ Chemist, Analytical Sciences Division, Mail Stop L-310, Lawrence Iivermore National Laboratory, Po Box 808, Iivermore, CA 94550 .

${ }^{2}$ Chemist, Nuclear Chemistry Division, Mail stop L-231, Lawrence Livermore National Laboratory, Po Box 808, Livermore, CA 94550 . 
ABSTRACT: For many years, Lawrence Livermore National Laboratory has collected monthly air-particulate filter samples from a variety of environmental monitoring stations on and off site. Historically the concentration and isotopic composition of uranium collected on these filters was determined by isotope dilution using a ${ }^{233} \mathrm{U}$ spike and thermal ionization mass spectrometry (TIMS). For samples containing as little as 10 nanograms of uranium, ICP-MS is now used to make these measurements to the required level of precision, about $5 \%$ in the measured $235 / 238$ and $233 / 238$. Unless particular care is taken to control bias in the mass filter, variable mass bias limits accuracy to a few percent. Measurements of the minor isotopes 236 (if present) and 234 are also possible and provide useful information for identifying the source of the uranium. The advantage of ICP-MS is in rapid analysis, $\approx 12$ minutes of instrument time per sample.

KEY WORDS: uranium, ICP-MS, isotopic ratio measurements, air filters, environmental monitoring 


\section{Introduction}

Like many other public and private sites, the Lawrence Livermore National Laboratory (LLNL) has an ongoing environmental monitoring program. This program includes the periodic collection of samples from a variety of media and locations. One aspect of this program is the determination of the concentration and isotopic composition of uranium collected by a series of air particulate samplers positioned to detect any significant concentration of particulate effluent from LLNL operations. These determinations involve an isotope dilution measurement of the uranium concentration based on adding a known quantity of high purity ${ }^{233} \mathrm{U}$ to the sample. The resulting ${ }^{233} \mathrm{U} /{ }^{238} \mathrm{U}$ ratio in the sample is then measured. The ${ }^{235} \mathrm{U} /{ }^{238} \mathrm{U}$ ratio is also measured to search for isotopically altered; ie, non-natural, uranium. Prior to 1988 , these determinations were made by thermal ionization mass spectrometry (TIMS). The purpose of this work was to show that inductively coupled plasma - mass spectrometry (ICP-MS) could more cost effectively make the measurements to the required level of precision and accuracy, about 5\%. While not part of the monitoring requirement and at less precision than is possible with TIMS, the ability to determine the minor uranium isotopes, ${ }^{234} \mathrm{U}$ and ${ }^{236} \mathrm{U}$, by ICP-MS has proven useful in characterizing samples that contain non-natural ${ }^{235} \mathrm{U} /{ }^{238} \mathrm{U}$ ratios.

The LINL environmental monitoring program, including details of air monitoring procedures and locations, has been described in a series of annual reports; eg, [1]. The work presented here will be restricted to monthly composite samples collected on the main 
LLNL site in the years 1988 through 1990. Filters were dissolved and the uranium extracted by acid digestion and ion exchange [2] before the samples were delivered to the authors. While the full chemical separation used is probably not necessary, a preliminary study showed that the dissolved solids to uranium ratio was too high to analyze the digested filters without some separation chemistry. Because the full separation chemistry was required for other determinations and our interest was in the capability of the ICP-MS for the isotopic measurements, no attempt was made in this work to minify the time involved in the chemical separations stage. It is of course possible that the ICP-MS could be used to determine other species of interest such as beryliium, lead, thorium, and plutonium without separating these elements from the uranium or each other.

\section{Experimental}

The measurement of isotope ratios in the authors' laboratory has been described previously $[3,4,5]$. Uranium aliquots from the separation chemistry were diluted to $15 \mathrm{~mL}$ with $0.1 \mathrm{~N} \mathrm{HNO}_{3}$ and aspirated into the ICP-MS at an uptake rate of approximately 0.6 $\mathrm{mL} / \mathrm{min}$. Two instruments, a VG PlasmaQuad and to a lesser extent a PQ2, were used in this work. For each sample ten sequential oneminute data accumulations were averaged together. The instruments were operated in the scanning mode. A typical accumulation was 1465 sweeps of 512 data channels covering the mass region 231.5$240.8 \mathrm{amu}$. For these conditions the dwell time per channel was 80 $\mu$ sec in each sweep. Separately from these measurements, the dead time of the electron multiplier was determined from measurements 
of the ${ }^{235} \mathrm{U} /{ }^{238} \mathrm{U}$ ratio in a solution of the National Institute of Standards and Technology (NIST) Standard Reference Material U005 [3]. Isotope ratios were calculated from peak areas where a fixed integration width of $0.9 \mathrm{amu}$ was used for each mass. To prevent sample-to-sample contamination, $0.1 \mathrm{~N} \mathrm{HNO}_{3}$ was aspirated between samples until the count rate at mass 238 , the most intense peak in all samples, dropped to background. For an initial set of 25 samples, the uranium aliquot was split and analyzed by both TIMS and ICP-MS.

At the beginning of a set of samples, a $10 \mathrm{ppb}$ solution of NIST U500 was analyzed to set the resolution and mass calibration and determine the mass bias. In addition to the U500 standard, this solution was spiked with high purity ${ }^{233} \mathrm{U}$ so that the three isotopes 233,235 , and 238 were in approximately equal abundance. This facilitated setting the resolution and mass calibration. (The $6 \times 10^{-4}$ effect of the spike on the $235 / 238$ ratio was ignored in making mass bias correction because it is much less than the precision of the data.) Figure 1 is a typical spectrum from a one minute data accumulation for this solution. The masses are baseline resolved and the continuum background is low. While the ${ }^{233} \mathrm{U} /{ }^{238} \mathrm{U}$ ratio in this solution was not known accurately, sampleto-sample reproducibility of the measurement provided a useful diagnostic. At the end of the analysis of the set of samples, typically 12-15, the spiked U500 was reanalyzed to establish the extent of drift in mass bias, resolution, and mass calibration. Typically the two analyses were averaged together to determine the mass bias to apply to the other samples. Because the signal 
in the ICP-MS is proportional to the elemental concentration, the U500 also provided a sensitivity calibration that was used to determine how many nanograms of uranium were in the sample analyzed and thereby the chemical yield of the separation chemistry.

Before analyzing samples, a $10 \mathrm{ppb}$ solution of NIST U010 was analyzed. This reference material was selected because its 234 and 235 abundances were similar to natural uranium. This standard was also spiked with ${ }^{233} \mathrm{U}$ to the level of $233 / 235 \approx 1$, which was in the range expected for the air filter samples. A typical spectrum for the spiked U010 is shown in Figure 2. While not shown, spectra of air filter samples were similar.

The integrated peak intensity data from all analyses for each group of samples were combined into an ASCII formatted file and imported into. a symphony [6] spreadsheet template for further data reduction. This spreadsheet was designed as a database with multiple selection criteria that automatically performed the data reduction steps required to calculate isotope ratios, prepare customer reports, and create a second, archival database of the final results for all samples. The intensity at mass 240 was taken as the instrumental background and subtracted from all other peaks. Isotope ratios were then calculated for each data accumulation. The measured ${ }^{235} \mathrm{U} /{ }^{238} \mathrm{U}$ ratios for the $\mathrm{U} 500$ were used to calculate a mass bias correction to be applied to all other samples and ratios according to the formula

$$
(235 / 238)_{\text {meas }}=(235 / 238)_{\text {true }} \times(1+\alpha)^{n} \approx(235 / 238)_{\text {true }} \times(1+n \alpha)
$$


where the "true" ratios are the accepted natural or standard values, "meas" indicates the measured value, $\alpha$ is the mass bias per amu, and " $n$ " is the amu difference in masses of the isotopes being "ratioed", three in this case. All other ratios were then corrected for mass bias using this formulation. The uncertainty on each isotope ratio for each sample was calculated as one standard deviation of the mean of the set of ten accumulations including the statistical uncertainty in the bias correction. The isotope ratios, their uncertainties based on the spread of the ten accumulations, the quantity of uranium in the measured sample, and the chemical yield were reported. The quantity of uranium on the filter, based on isotope dilution, was calculated by the end users of the data and is not of concern in discussion of the accuracy and precision of the isotope ratio measurements. Results

\section{Standards}

To establish that ICP-MS could be used in place of TIMS for the determination of ${ }^{235} \mathrm{U} /{ }^{238} \mathrm{U}$ and ${ }^{233} \mathrm{U} /{ }^{238} \mathrm{U}$ in air filter samples, a set of samples was measured by both techniques. In Figure 3 , the ratios of the $235 / 238$ ratios observed by ICP-MS to those observed by TIMS have been plotted as a function of the amount of uranium in the aliquots analyzed by ICP-MS. Over three orders of magnitude in uranium content, there is no clear bias between the methods and the methods typically agree within $3 \%$, which is well within the objective of $5 \%$. Adequate accuracy was obtained for samples containing <10 ng of uranium; ie, a concentration of $<1$ $\mathrm{ppb}$ in the measured solution. For the ICP-MS, the standard 
deviations of the means for the various analyses ranged from $1.5 \%$ to $0.02 \%$ depending on the ratio and sample size. The TIMS uncertainties were somewhat smaller. While the scatter in Figure 1 is acceptable for the objectives of the work, it is somewhat larger than the uncertainties estimated for the analyses.

Figure 4 is a similar plot for the 233/238 measurements. In this case, there is a bias of somewhat more than $2 \%$ between the methods with ICP-MS giving the lower values. Given the scatter in the data, it is difficult to establish whether there is a trend with the amount of uranium, but there is no evidence that the use of larger samples would reduce the bias. Although not shown, the ratio of ICP-MS to TIMS results for $233 / 238$ shows no trend as a function of the magnitude of the measured ratio over the range of this data set, $0.001-0.15$. The origin of this bias is unclear and while disturbing, it was judged to be inconsequential for these measurements.

Although the initial objective of this work was to measure ${ }^{233} \mathrm{U} /{ }^{238} \mathrm{U}$ and ${ }^{235} \mathrm{U} /{ }^{238} \mathrm{U}$ ratios and to do so in a "production mode", variations in ${ }^{235} \mathrm{U} /{ }^{238} \mathrm{U}$ alone are not sufficient to isotopically characterize the materials causing the variation. Determination of the abundances of the minor isotopes ${ }^{234} \mathrm{U}$ and ${ }^{236} \mathrm{U}$ allows one to distinguish among the possible sources of contamination. These minor isotopes can be precisely measured by TIMS but such measurements were not emphasized in the previous air particulate studies at LLNL. When the TIMS to ICP-MS study just discussed was undertaken, the sensitivity of the ICP-MS was not sufficient to measure the minor isotopes to a useful precision. In addition 
those isotopes were not measured in the TIMS study so no direct comparison between the techniques is possible. Shortly after this work began, the sensitivity of the ICP-MS was increased to the point where the minor isotopes could be measured to a useful level of precision, $<10 \%$, within the normal data scans. In that no additional effort or operating time was involved in making these measurements, they were added to the normally reported data set. As will be shown below, they have proven to be quite useful. The long term reproducibility of multiple measurements of the ${ }^{233} \mathrm{U}$ spiked $\mathrm{U} 500$ and U010 standards can be used to access data quality and limitations. These data are summarized in Table 1 . If the data are normally distributed, the standard deviation of the distribution of measurements for a particular isotope ratio and standard should be of approximately the same magnitude as the average standard deviation of the mean of the ten accumulations in a single analysis. For the three-year data set, the data clearly scatter more than predicted by a single analysis. This is particularly true of the $233 / 238$ values. For the 1990 data taken alone, the scatter is reduced, particularly for the 233/238 ratios, but the scatter is still generally greater than expected.

A closer examination of the data for the individual analyses revealed two effects that degrade data quality. First, in the 1988-1989 data, which were all collected on the same instrument, there was a distinct change in mass bias during the course of each group of analyses as measured by the U500 standard measurements at the beginning and end of the data sets. In that the average of the two $\mathrm{U} 500$ analyses was used to correct the 
other samples for mass bias, this manifests itself in the bias corrected $233 / 238$ ratio of the early analysis of each set being typically $3 \%$ higher than the latter. Consistent with this observation,-plots of 233/238 and 235/238 for the U010 standard, which were analyzed shortly after the first U500 of each set, show correlations of increasing "bias corrected" ratios with increasing bias correction. Near the end of 1989, this variability in bias during sets of analyses disappeared and analysis-to-analysis scatter was substantially reduced. Unfortunately, we have not been able to determine the cause of the variability or why it changed.

The second effect limiting data quality, particularly in the 1988-1989 period, appears to be a bias affecting mass 233 that does not follow equation (1). The distribution of $233 / 238$ measurements has a standard deviation of nearly $2 \%$ for each of the standards. This is three times the spread of the $235 / 238$ values for U010. While one would expect a somewhat larger spread in $233 / 238$ because of the extrapolation of the mass bias correction from three mass units to five, a factor of three is not expected. If one calculates the $233 / 235$ values from the raw data rather than from the $235 / 238$ and $233 / 238$ ratios, the spread in $233 / 235$ is comparable to that of the $233 / 238$ ratios, but in the 233/235 case, the mass bias correction is applied over only a difference of two masses. If the bias law of equation 1 or any similar law based on mass difference applies, then the 233/235 ratios should show less scatter due to the correction than the $235 / 238$ ratios. Based on the observed scatter, we conclude that 
an additional, unidentified mechanism, perhaps associated with variable quadrupole transmission, was limiting the precision of the 233 measurements in the 1988-1989 time period.

\section{Air Filters}

In Figure 5, the observed $235 / 238$ ratios for nearly 300 particulate air filter samples are plotted in the order they were analyzed. The vast majority have near natural composition, 235/238=0.0072. (Of course a component enriched in 235 could be mixed with one depleted in 235 to give the natural value.) only two samples show significant enrichment in 235 . While there is no basis to discount these points, which occurred within one month's data set, their ion yields are too low to use the minor isotopes 234 and 236 to help establish the origin of the apparent enrichment. There are two groups of samples which show distinct depletions in the $235 / 238$ ratio. For these samples, sufficient ions were collected to allow one to characterize the isotopic composition of the uranium component responsible for the depletion.

In Figure 6 the $234 / 238$ ratio for each filter with more than one million 238 ions per accumulation has been plotted against the $235 / 238$ ratio. All the samples with depleted $235 / 238$ show a strongly correlated depletion in 234/238, and samples from both incidences of observed depletion follow the same trend. Furthermore, the regression line fit to these points projects toward the uranium composition commonly known as D38. D38 is the residual material, "tails", of uranium which has been processed through a reactor and then subjected to isotope separation to 
recover ${ }^{235} \mathrm{U}$. In such a plot, the position of a point between two components, natural and $\mathrm{D} 38$ in this case, is a measure of the relative amounts of the components. The samples most depleted in 235 contain three parts of D38 to every part of natural uranium. One might expect these samples to contain unusually high concentrations of uranium, but this is not the case. This may be related to the fact that both occurrences of depleted uranium occurred in winter months when it rains in Livermore and there is less dust in the air.

Looking at Figure 6 in more detail, one sees that the points clustered near the natural isotopic composition show a slight offset from the natural $234 / 238$ value. The cause of this displacement of $\approx 7 \times 10^{-6}$ is unknown, but the fact that the meaningful conclusions can be based on the observation of only a few hundred ions of the minor isotope argues favorably for the technique. If the U500 or U010 standards had contaminated the instrument, sample points would be displaced in the directions indicated by the lines emanating from the natural point. In this discussion two points, circled in the figure, have been excluded from the correlation because their 236 values were anomalous, see below.

Figure 7 is similar to Figure 6 except the $236 / 238$ ratio has been plotted. Mass 236 is particularly sensitive to the presence of isotopically non-natural material because this isotope does not occur in nature. Again the samples depleted in 235 strongly trend toward D38. In this case the non-depleted samples cluster around the natural value of $236 / 238=0$, and again there is no 
evidence of $\mathrm{U} 500$ or U010 contamination of the non-depleted samples. The circled sample and another which falls above the boundary of the plot have been excluded in fitting the correlation line. They are the same samples excluded in Figure 6 . It is possible that these points represent a three component mixture of natural, 235-depleted, and U010-like compositions, but with so little data such a conclusion would be tenuous.

\section{Conclusion}

ICP-MS has been shown to be a useful tool for the routine determination of uranium isotopic composition in particulate air. filter samples used for environmental monitoring. All isotopes of uranium can be determine to a useful level of precision within roughly 12 minutes of instrument time. Measurements of the minor isotopes provide a powerful method of characterizing the composition and potential sources of isotopically non-natural uranium. While ICP-MS is not as precise as TIMS, the ease of sample introduction, rapid data collection including minor isotopes, and overall system simplicity make ICP-MS the method of choice for such determinations. For more precise determinations of isotope ratios, one must pay particular attention to mass bias effects.

\section{Acknowledgement}

We thank Donald Macqueen, Philip Miller, and Patrick Epperson for helping us organize our thoughts on the scatter of the standards' data and Gerald Hunt for his contributions to hardware and software improvements. This work was performed under the auspices of the U.S. Department of Energy by Lawrence Livermore National Laboratory under contract No. W-7405-ENG-48. 


\section{References}

[1] Wilt, Gloria C. (ed.), "Environmental Report 1992", Lawrence Livermore National Laboratory report UCRL-50027-92, Livermore, CA.

[2] Garrison, J., MacLean, S.,Bocanegra, R.,Ruggieri, M.R., and Buddemeier, R.W., "Radiochemical Methods of the site Environmental Monitoring Program", Document ID M-122,Rev.1, January 1985, Lawrence Livermore National Laboratory, Livermore, CA.

[3] Russ III, G. Price and Bazan, J.M., "Isotopic ratio measurements with an inductively coupled plasma source mass spectrometer", Spectrochimica Acta 42B, 1987, pp 49-62. [4] Russ III, G. Price, "Isotope ratio measurements using ICPMS", in A.R. Date and A.L. Gray (ed.), Applications of ICP-MS, Blackie and Son, Glasgow, 1988, pp 90-114.

[5] Russ III, G. Price, Bazan, Jeanne M., and Date, Alan R., "Osmium isotopic ratio measurements by inductively coupled plasma source mass spectrometry, Analytical Chemistry 59, 1987, pp 984989.

[6] "Symphony", Lotus Development Corp, Cambridge, Massachusetts. 
Table 1--Reproducibility of standards

\section{$233 / 238 \quad 234 / 238^{\mathrm{a}} \quad 235 / 238 \quad 236 / 238 \quad$ Analyses}

\section{Spiked 0500}

\begin{tabular}{|c|c|c|c|c|}
\hline NIST & $\cdots$ & 0.01042 & $0.9997^{b}$ & 0.00152 \\
\hline Avg $1 \sigma_{m}{ }^{c}$ & 0.002 & 0.00005 & 0.0015 & 0.00002 \\
\hline $\begin{array}{c}\operatorname{Avg}(1988-1990) \\
1 \sigma\end{array}$ & $\begin{array}{r}0.913 \\
18\end{array}$ & $\begin{array}{r}0.01072 \\
25\end{array}$ & $\ldots{ }^{d}$ & $\begin{array}{r}0.00154 \\
3\end{array}$ \\
\hline $\begin{array}{c}\operatorname{Avg}(1990) \\
1 \sigma\end{array}$ & $\begin{array}{r}0.902 \\
5\end{array}$ & $\begin{array}{r}0.01049 \\
9\end{array}$ & $\cdots$ & $\begin{array}{r}0.00155 \\
2\end{array}$ \\
\hline
\end{tabular}

\section{Spiked 0010}

$\begin{array}{lllll}\text { NIST } & \ldots & 0.000055 & 0.01014 & 0.000069\end{array}$

$\begin{array}{lrrrr}\text { Avg } 1 \sigma_{\mathrm{m}}{ }^{c} & 0.00004 & 0.000003 & 0.00004 & 0.000003 \\ \operatorname{Avg}(1988-1990) & 0.01000 & 0.000057 & 0.01018 & 0.000070 \\ 1 \sigma & 17 & 5 & 6 & 6\end{array}$

$\operatorname{Avg}(1990)$

$$
\begin{array}{rrrr}
0.00986 & 0.000054 & 0.01016 & 0.000070 \\
6 & 4 & 6 & 5
\end{array}
$$

asample data for mass 234 have had the ${ }^{233} \mathrm{U}$ spike's contribution of $234 / 233=1.8 \times 10^{-4}$ subtracted from the reported values.

'The spike's contribution to 235 and 238 which lowers the true ratio to 0.9991 in this standard was ignored because it is much less than the precision of the measurement.

"Uncertainty of the mean for a single analysis.

'Used to normalized for mass bias. 


\section{Figure captions}

Fig. 1 Typical spectrum for spiked U500 at $10 \mathrm{ppb}$.

Fig. 2 Typical spectrum for spiked U010 at $10 \mathrm{ppb}$.

Fig. 3 Comparison of ICP-MS and TIMS $235 / 238$ results for 25

air filter samples.

Fig. 4 Comparison of ICP-MS and TIMS $233 / 238$ results for the same 25 air filter samples as in Figure 3 .

Fig. $5 \quad{ }^{235} \mathrm{U} /{ }^{238} \mathrm{U}$ for particulate air samplers. Note data have been segregated on the basis of signal strength. Only the higher intensity samples have sufficient signal for further evaluation involving the minor isotopes.

Fig.6 Three isotope correlation plot, ${ }^{234} \mathrm{U} /{ }^{238} \mathrm{U}$ vs. ${ }^{235} \mathrm{U} /{ }^{238} \mathrm{U}$, for the higher signal samples from Fig. 5. D38 is shown as a field rather than a point because its isotopic composition varies depending on the history of the material.

Fig. 7 Three isotope correlation plot, ${ }^{236} \mathrm{U} /{ }^{238} \mathrm{U}$ vs. ${ }^{235} \mathrm{U} /{ }^{238} \mathrm{U}$, for the samples plotted in Fig. 6 . 
TYPICAL SPIKED U500 STANDARD

(1 min data accumulation)

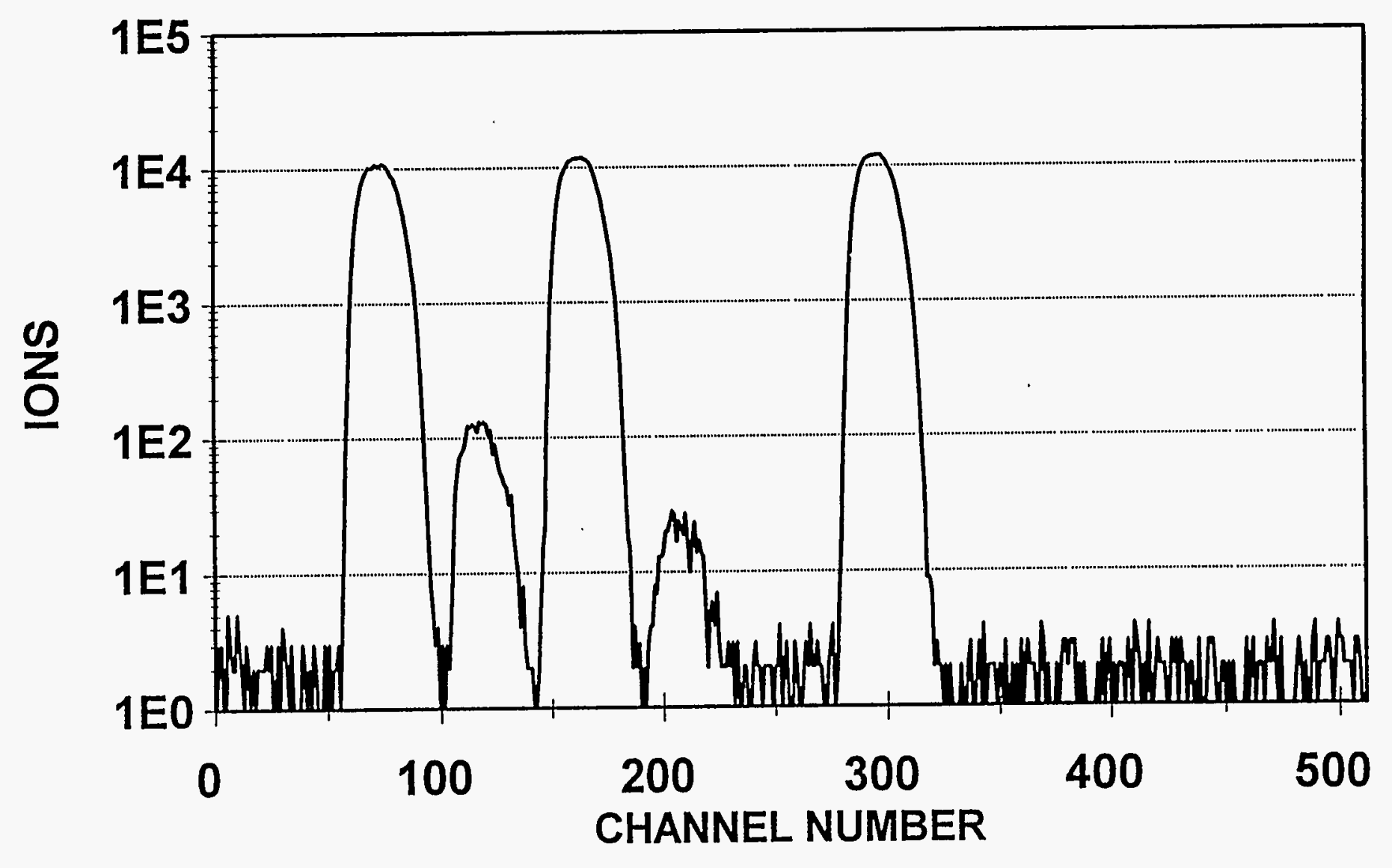

Fif 1 Russ $x$ Bazan. 


\section{TYPICAL SPIKED U010 STANDARD}

(1 min. data accumulation)

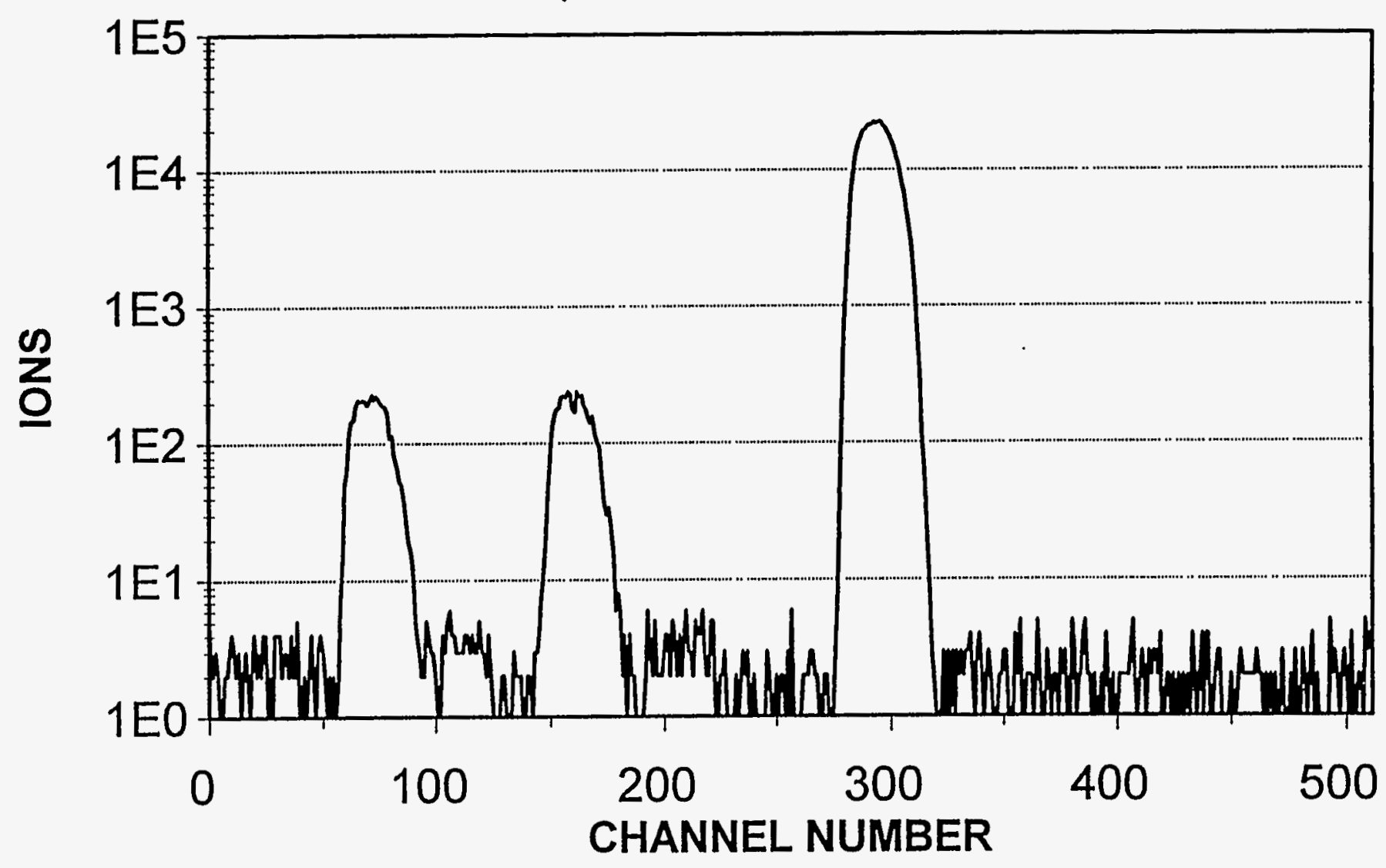

Fis. 2 Russ $*$ Bazan 


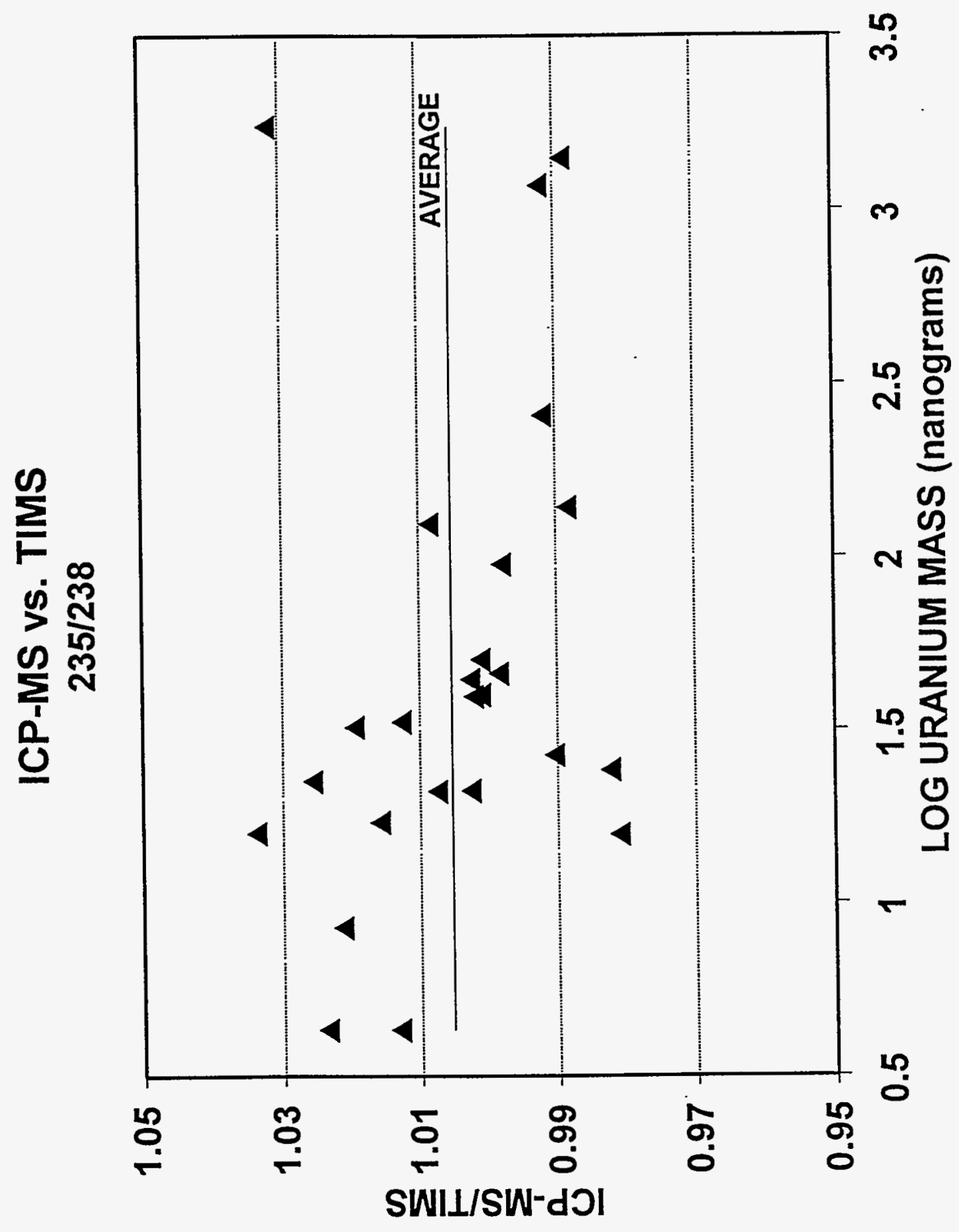




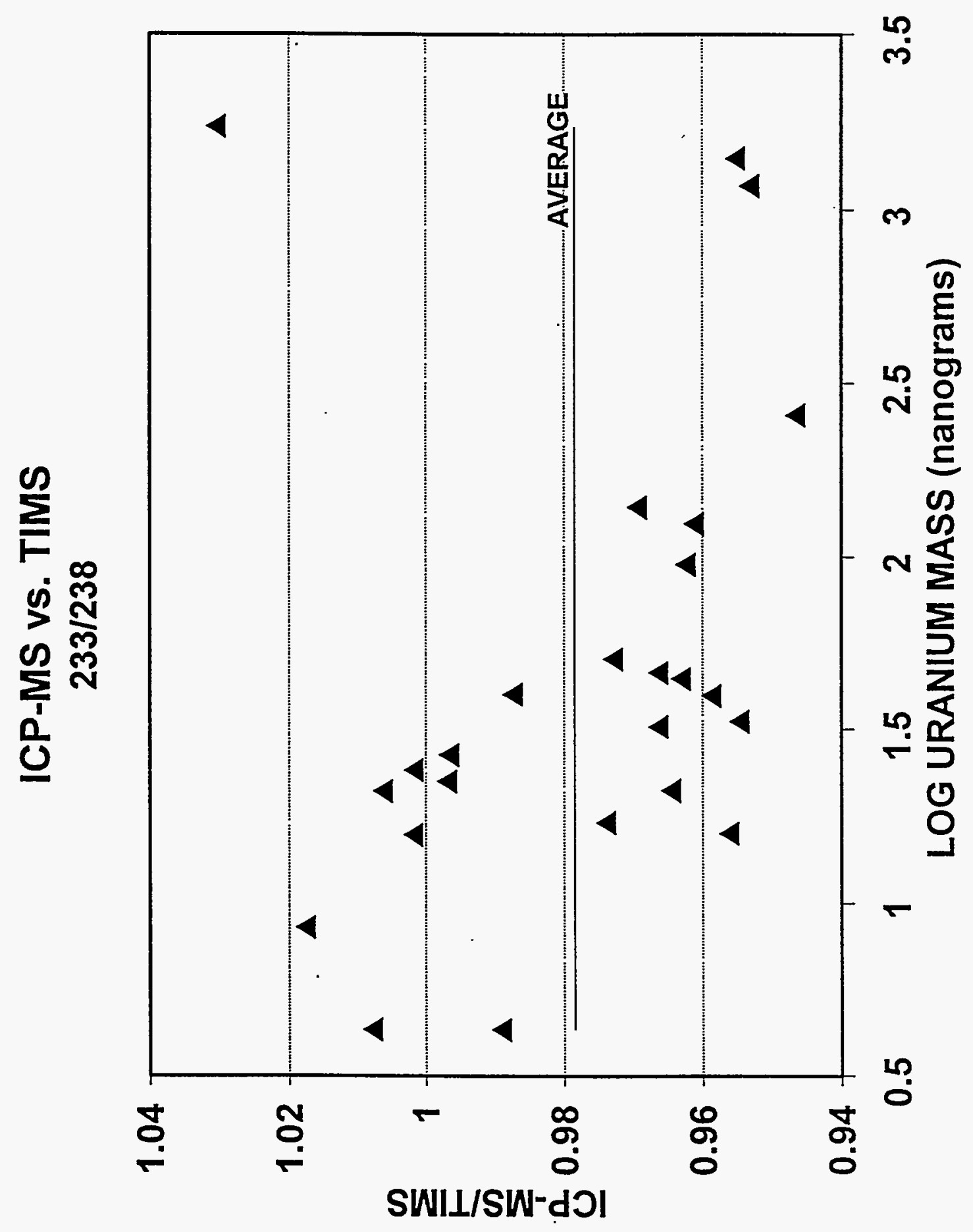




\section{Air Filters (1988-1990)}

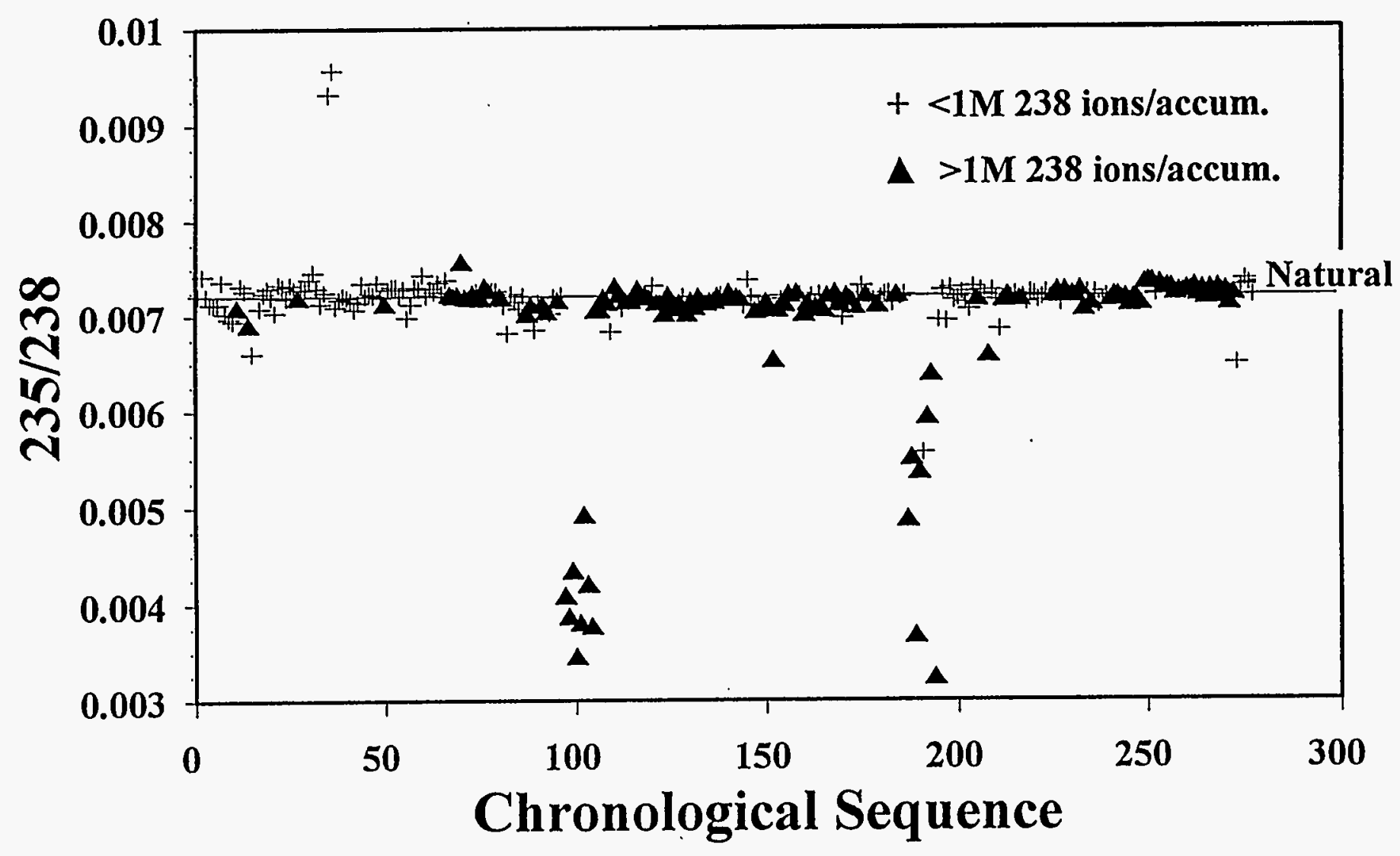

Fys Russur Bugen 


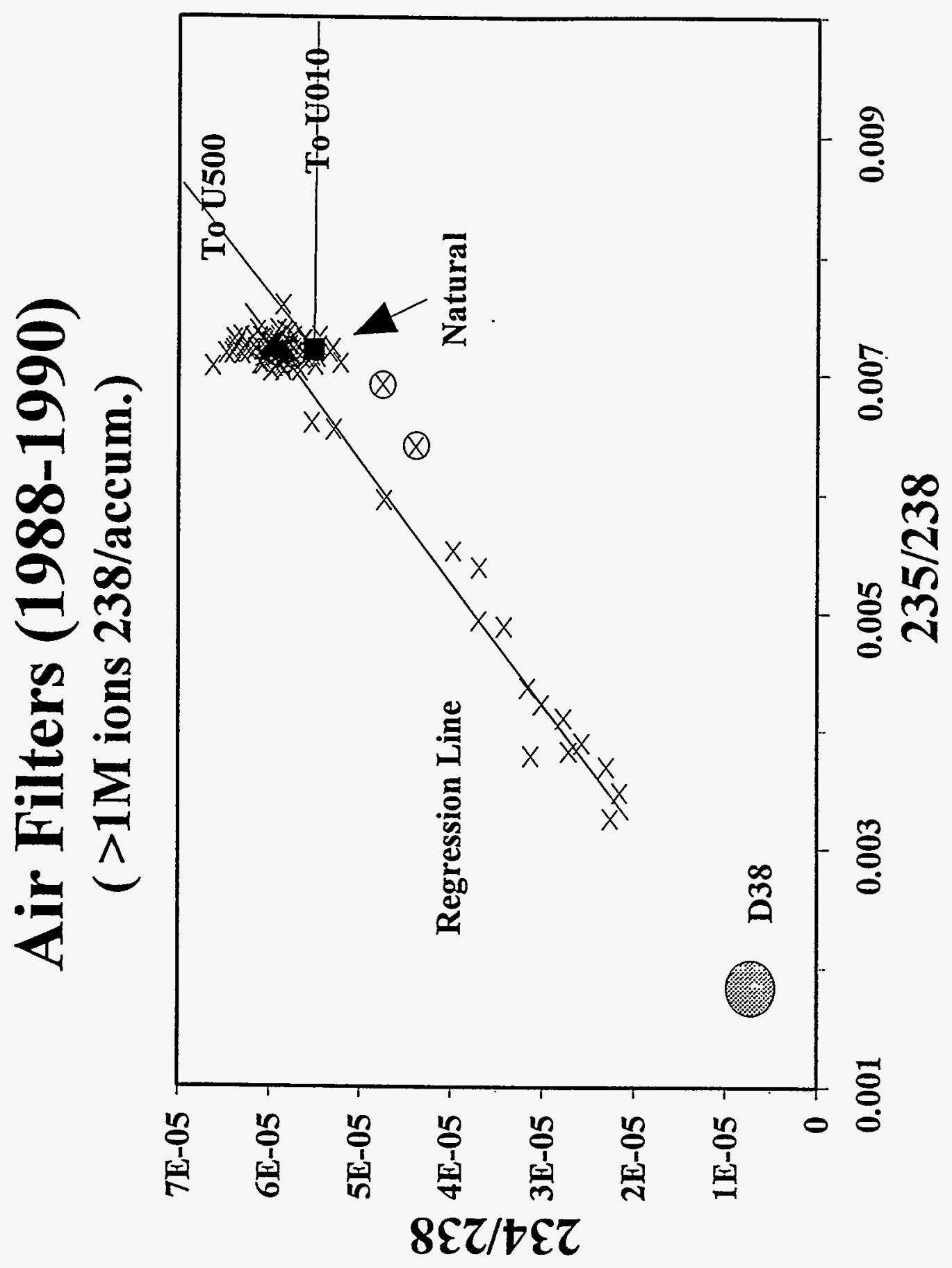




\section{Air Filters (1988-1990) ( $>1 \mathrm{M}$ ions 238/accum.)}

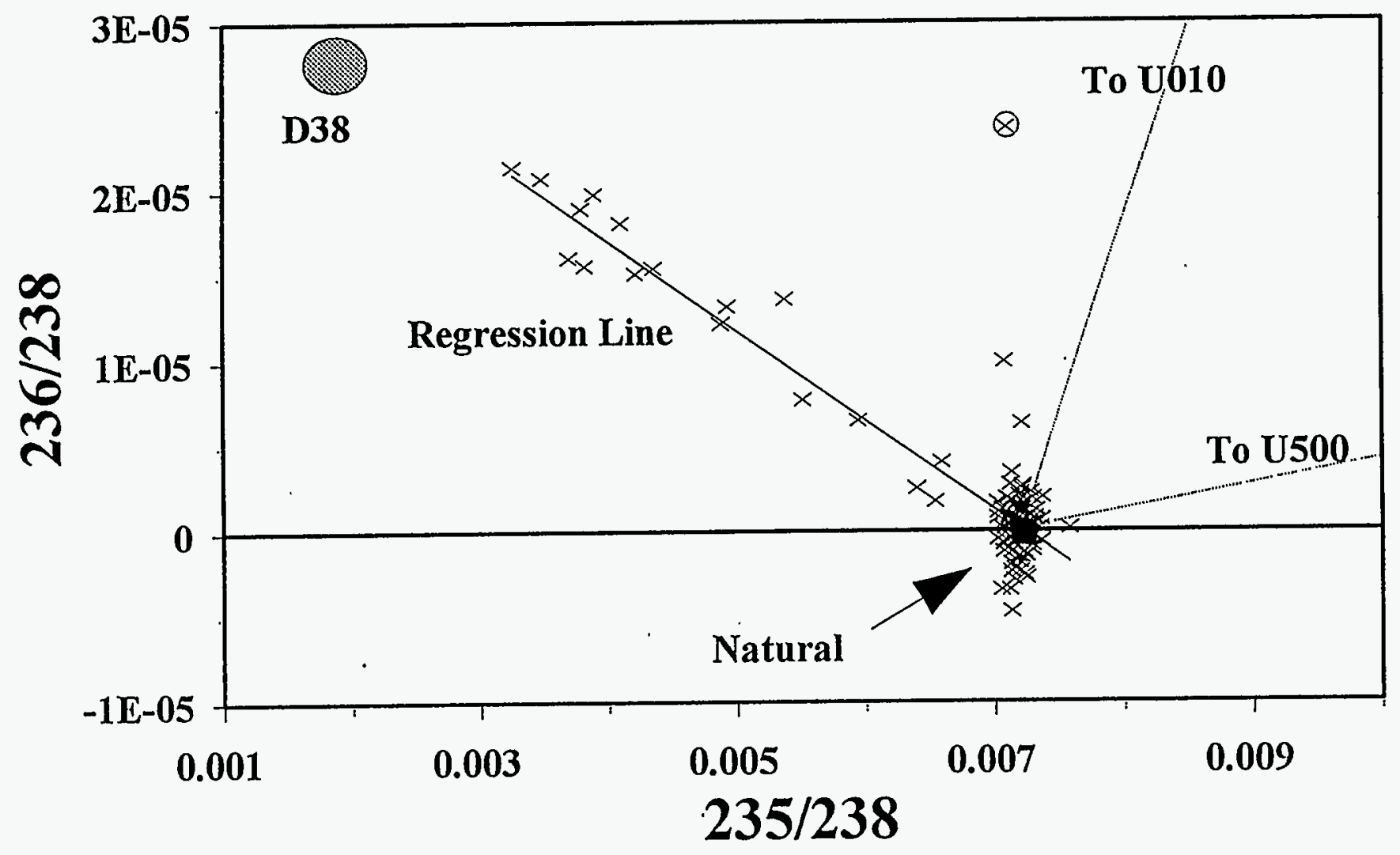

F.g 7 Russt Brzan 